\title{
El tiempo de llene capilar prolongado es predictor de una saturación venosa central de oxígeno disminuida
}

\author{
R. Bustos • O. Padilla
}

\begin{abstract}
A prolonged capillary refill is predictor of low central venous oxygen saturation

Introduction: Educational programs in pediatric life support endorse a capillary refill time $>2 \mathrm{~s}$ as an indicator of shock. In the emergency room, a barrier to the implementation of an early goal directed therapy, aiming at central venous oxygen saturation $\left(\mathrm{ScvO}_{2}\right) \geq 70 \%$ is the insertion of central venous catheter (CVC). Objective: To establish the predictive value of capillary refill time $>2 \mathrm{~s}$ to detect $\mathrm{ScvO}_{2}<70 \%$ in children admitted to Intensive Care Units. Patients and Method: Prospective study. We included 48 children admitted in the first 24 hours in ICU with superior vena cava CVC. Simultaneously, we measured $\mathrm{ScvO}_{2}$ and capillary refill time in the heel of upper extremity or toe. Results: There were 75 paired measurements $\mathrm{ScvO}_{2}(75,9 \pm 8,4 \%)$ and capillary refill capillary $(1,9 \pm 1,0 \mathrm{~s})$. We found an inverse correlation between capillary refill time and $\mathrm{ScvO}_{2}$ $(\mathrm{r}-0,58)$. The ROC curve analysis revealed an excellent ability for the capillary fill time $>2 \mathrm{~s}$ to predict $\mathrm{ScvO}_{2}$ $<70 \%$ (AUC 0,94) $(95 \%$ CI 0,87-0,98). Conclusions: A prolonged capillary refill time $>2 \mathrm{~s}$, is a predictor of $\mathrm{ScvO}_{2}<70 \%$ in children admitted to ICU, which supports the current recommendations. This finding may be relevant in emergency units where the use of CVC is limited and $\mathrm{ScvO}_{2}$ is not available.

(Key words: Capillary refill time, central venous oxygen saturation, pediatrics, early goal directed therapy).

Rev Chil Pediatr 2014; 85 (5): 539-545
\end{abstract}

\section{RESUMEN}

Introducción: Programas educativos de reanimación pediátrica establecen que un tiempo de llene capilar $>2 \mathrm{~s}$ es un indicador de shock. En unidades de emergencia, una barrera para la implementación de una reanimación precoz guiada por metas, teniendo como objetivo una saturación venosa central de oxígeno $\left(\mathrm{ScvO}_{2}\right) \geq 70 \%$, es la inserción de un catéter venoso central (CVC). Objetivo: Determinar el valor predictivo de un tiempo de llene capilar $>2 \mathrm{~s}$ en la detección de $\mathrm{ScvO}_{2}<70 \%$ en niños ingresados a la Unidad de Cuidados Intensivos. Pacientes y Método: Estudio prospectivo. Se incluyeron 48 niños ingresados en las primeras $24 \mathrm{~h}$ en UCI con CVC en la vena cava superior. De manera simultánea se determinaron $\mathrm{ScvO}_{2}$ y tiempo de llene capilar en talón o dedo de extremidad superior. Resultados: Se obtuvieron 75 mediciones pareadas de $\mathrm{ScvO}_{2}(75,9 \pm 8,4 \%)$ y llene capilar $(1,9 \pm 1,0 \mathrm{~s})$, observándose una correlación inversa entre llene capilar y $\mathrm{ScvO}_{2}(\mathrm{r}=-0,58)$. El análisis de la curva ROC reveló una excelente capacidad del tiempo de llene capilar $>2 \mathrm{~s}$ para predecir una $\mathrm{ScvO}_{2}$

Recibido el 16 de junio de 2013, última versión aceptada para publicación el 25 de mayo de 2014.

Raúl Bustos B. $(\bowtie)$

Unidad de Medicina Intensiva Pediatrica, Hospital Guillermo Grant Benavente, Concepción.

E-mail: robustos64@yahoo.com.ar

Oslando Padilla P.

Departamento de Salud Pública, Pontificia Universidad Católica de Chile. 
$<70 \%$ (AUC $=0,94$, IC 95\% = 0,87-0,98). Conclusiones: La prolongación del tiempo de llene capilar $>2 \mathrm{~s}$ es predictor de $\mathrm{ScvO}_{2}<70 \%$ en niños críticamente enfermos. Este hallazgo apoya las recomendaciones actuales y podría ser relevante en unidades de emergencia donde el uso de $\mathrm{CVC}$ es limitado y la $\mathrm{ScvO}_{2}$ no está disponible. (Palabras clave: Tiempo de llene capilar, saturación venosa central de oxígeno, pediatría, reanimación). Rev Chil Pediatr 2014; 85 (5): 539-545

\section{Introducción}

Actualmente es aceptado que una disminución de la saturación venosa central de oxígeno $\left(\mathrm{ScvO}_{2}\right)$, obtenida a través de un catéter venoso central (CVC) en el territorio de la vena cava superior, puede revelar un desequilibrio entre el aporte y las demandas de oxígeno, y por lo tanto, disoxia tisular.

En unidades de emergencia, intervenciones hemodinámicas basadas en la normalización de $\mathrm{ScvO}_{2}$ a valores $\geq 70 \%$-reanimación precoz guiada por metas (early goal directed therapy) - han logrado disminuir la mortalidad en pacientes adultos sépticos ${ }^{1}$. Estos protocolos y paquetes de medidas han sido adoptados por la "Campaña Sobreviviendo a la Sepsis" y son recomendados por el American College of Critical Care Medicine en las guías de tratamiento del shock séptico pediátrico ${ }^{2}$.

En general, cuando estas estrategias se han implementado en instituciones de salud, se ha observado una adherencia y cumplimiento menores a un $50 \%$. Así, una de las principales barreras para la implementación de estos paquetes de medidas son el tiempo requerido y la dificultad técnica en insertar un CVC en sala de emergencia ${ }^{3-5}$.

Numerosos grupos y programas educativos, incluida la Organización Mundial de la Salud, la Academia Americana de Pediatría, a través del curso Advanced Pediatric Life Support (APLS), la American Heart Association, a través del curso Pediatric Advanced Life Support (PALS) avalan el uso del tiempo de llene capilar (TLLC) como una importante herramienta clínica para identificar aquellos pacientes en riesgo de shock circulatorio ${ }^{6-7}$. Las guías del PALS y APLS establecen un tiempo de llene capilar $>2$ s como indicador de shock y hacen énfasis en que una de la metas clínicas de la reanimación es su normalización a valores $\leq 2$ s.

El objetivo de esta investigación es determinar el valor predictivo de un TLLC $>2 \mathrm{~s}$ en la detección de $\mathrm{ScvO}_{2}<70 \%$ en niños ingresados a la Unidad de Cuidados Intensivos (UCI).

\section{Pacientes y Método}

\section{Diseño}

Estudio prospectivo observacional realizado en la UCI pediátrica del Hospital Guillermo Grant Benavente de Concepción, aprobado por el Comité de Ética clínico de nuestro hospital. Esta UCI tiene 8 camas y admite pacientes de 1 mes a 15 años con todo tipo de patología médico quirúrgica a excepción de post operados de cirugía cardíaca. Durante el período de estudio de 12 meses (febrero 2009-febrero 2010), previo consentimiento informado, se incluyeron 48 niños ingresados en las primeras $24 \mathrm{~h}$ con CVC. En todos los pacientes se insertaron catéteres por vía yugular interna, siendo verificada su correcta localización con una radiografía de tórax.

Se excluyeron pacientes con cardiopatías congénitas cianóticas, anemia (hemoglobina $<10 \mathrm{~g} / \mathrm{dL}$ ) y fiebre (temperatura $\left(\mathrm{T}^{\circ}\right)>38,5^{\circ} \mathrm{C}$ ) durante la medición del TLLC y toma de la muestra sanguínea.

\section{Procedimientos}

El TLLC se definió como el tiempo que toma un lecho capilar distal en obtener su coloración después hayan sido vaciados por la aplicación de presión.

El TLLC fue realizado por la enfermera a cargo del paciente, con la ayuda de un cronómetro y presionando durante $3 \mathrm{~s}$ el talón o dedo de extremidad superior, manteniendo la extremidad sobre el nivel del corazón para evi- 
tar el efecto del llene venoso. Los valores obtenidos de TLLC fueron aproximados a enteros cercanos. De manera simultánea se determinó la $\mathrm{ScvO}_{2}$ por el CVC. Las muestras sanguíneas fueron procesadas en un analizador de gases Cobas Ob 221 (Roche Diagnostics).

\section{Análisis estadístico}

Los datos continuos se presentan mediante mínimo, máximo, percentiles, medias y desviación estándar. Los datos categóricos se expresan en porcentaje. Se calculó correlación no paramétrica de Spearman entre la $\mathrm{ScvO}_{2}$ y el TLLC. Se generó una curva receiver operator characteristic (ROC) para evaluar el valor predictivo del TLLC en determinar $\mathrm{ScvO}_{2}<70 \%$. A partir de un valor del TLLC $>2 \mathrm{~s}$, se realizó un análisis de sensibilidad y la especificidad, valores predictivos y razón de verisimilitud para la detección de $\mathrm{ScvO}_{2}<70 \%$. Estos datos se presentan con sus intervalos de confianza (IC) al 95\%. Los análisis se realizaron en el programa SPSS, versión 17. Un valor $p<0,05$ fue considerado como estadísticamente significativo.

\section{Resultados}

Se realizaron setenta y cinco mediciones pareadas de $\mathrm{ScvO}_{2}$ y TLLC en 48 pacientes. Las características demográficas y diagnósticos de ingreso aparecen en la tabla 1 . Seis $(12,5 \%)$ de los ingresos fueron electivos. Veintitrés $(48 \%)$ fueron pacientes con criterios de shock séptico y que requirieron drogas vasoactivas y 37 (77\%) se encontraban en ventilación mecánica durante la medición. Los valores obtenidos de $\mathrm{ScvO}_{2}$ y TLLC fueron 75,9 $\pm 8,4 \%$ y $1,9 \pm 1,0 \mathrm{~s}$, respectivamente. Dieciséis de las mediciones de $\mathrm{ScvO}_{2}$ fueron inferiores a 70\% y tres menores a $60 \%$. Al momento de la determinación del TLLC, el lactato fue de 2,2 $\pm 2,0$ $\mathrm{mmol} / \mathrm{L}$, el hematocrito de $31,3 \pm 1 \%$ y la $\mathrm{T}^{\circ}$ de los pacientes fue de $37,3 \pm 0,6^{\circ} \mathrm{C}$.

El análisis de la curva ROC reveló una capacidad excelente del TLLC $>2 \mathrm{~s}$ para predecir $\mathrm{ScvO}_{2}<70 \%$, con un área bajo la curva de 0,94 (IC 95\% 0,87-0,98, p = 0,0001, figura 1). El TLLC $>2$ s presentó una sensibilidad de $86,7 \%$ (IC 95\% 69-100) y una especificidad de $91,7 \%$ (IC 95\% 85,7-99,4) para detectar $\mathrm{ScvO}_{2}<70 \%$. La razón de verosimilitud positiva fue de 10 con un valor predictivo positivo de 72 (tabla 2). La correlación no paramé-

Tabla 1. Características demográficas y clínicas de los pacientes incluidos en el estudio

\begin{tabular}{|lc|}
\hline & n: $\mathbf{4 8}$ \\
\hline Edad meses & $74 \pm 60$ \\
Género M/F & $30 / 18$ \\
PRISM & $19 \pm 10$ \\
Diagnósticos & \\
Neumonía & 18 \\
Politraumatismo & 8 \\
Meningitis-meningococcemia & 6 \\
Peritonitis & 5 \\
Gran quemado & 4 \\
Leucemia & 3 \\
Otros & 4 \\
Shock séptico $\mathbf{n}(\%)$ & $23(48)$ \\
Uso drogas vasoactivas $\mathbf{n}$ (\%) & $23(48)$ \\
Norepinefrina & 18 \\
Dopamina & 5 \\
Dobutamina & 10 \\
Uso de VM $\mathbf{n}$ (\%) & $37(77)$ \\
Mortalidad $\mathbf{n}$ (\%) & $5(10)$ \\
\hline
\end{tabular}

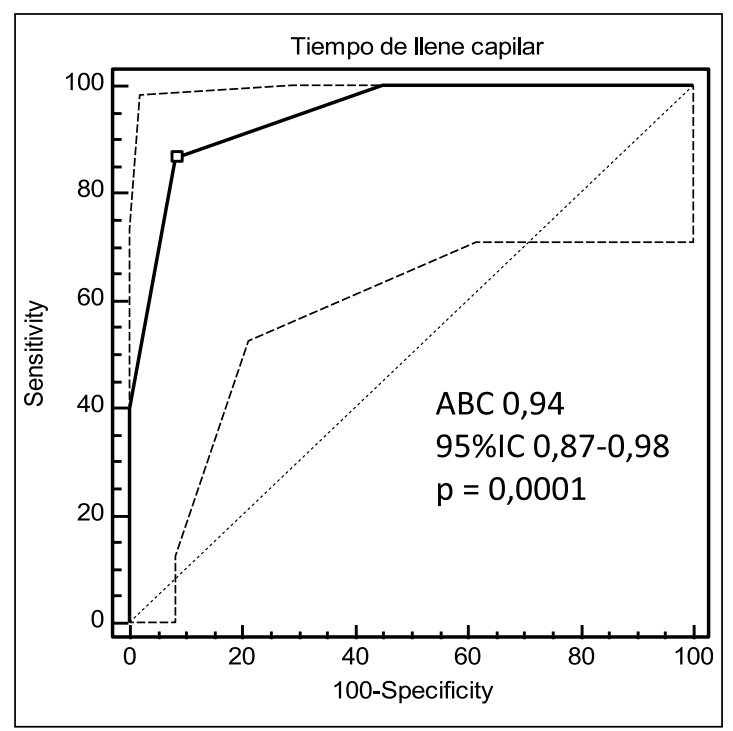

Figura 1. Análisis de la curva receiver operator characteristic (ROC). Demuestra la capacidad predictiva del tiempo de llene capilar para detectar $\mathrm{ScvO}_{2} . \leq 70 \%$. ABC: Área bajo la curva. IC: Intervalo de confianza. 
Tabla 2. Sensibilidad, especificidad, valor predictivo positivo y negativo para el tiempo de llene capilar

\begin{tabular}{|c|c|c|c|c|c|c|}
\hline Llene capilar seg & $\begin{array}{c}\text { Sens. } \\
95 \% \text { IC }\end{array}$ & $\begin{array}{l}\text { Espec. } \\
95 \% \text { IC }\end{array}$ & $\mathbf{R V}+$ & RV- & VP+ & VP- \\
\hline$>1$ & $\begin{array}{c}100 \\
78-100\end{array}$ & $\begin{array}{c}55 \\
42-68\end{array}$ & 2,22 & 0,00 & 35,7 & 100,0 \\
\hline$>2^{*}$ & $\begin{array}{c}86,67 \\
(60-98)\end{array}$ & $\begin{array}{c}91,67 \\
(82-97)\end{array}$ & 10,40 & 0,15 & 72,2 & 96,5 \\
\hline$>3$ & $\begin{array}{c}40 \\
16-68\end{array}$ & $\begin{array}{c}100 \\
94-100\end{array}$ & & 0,60 & 100 & 87,0 \\
\hline$>4$ & $\begin{array}{c}6,67 \\
1-32\end{array}$ & $\begin{array}{c}100 \\
94-100\end{array}$ & & 0,93 & 100,0 & 81,1 \\
\hline$>5$ & $0-22$ & $\begin{array}{c}100 \\
94-100\end{array}$ & & 1,00 & & 80,0 \\
\hline
\end{tabular}

Sens: sensibilidad. Espec: especificidad .VP+: valor predictivo positivo .VP -: valor predictivo negativo. RV +: razón de verosimilitud positiva .RV -: razón de verosimilitud negativa.

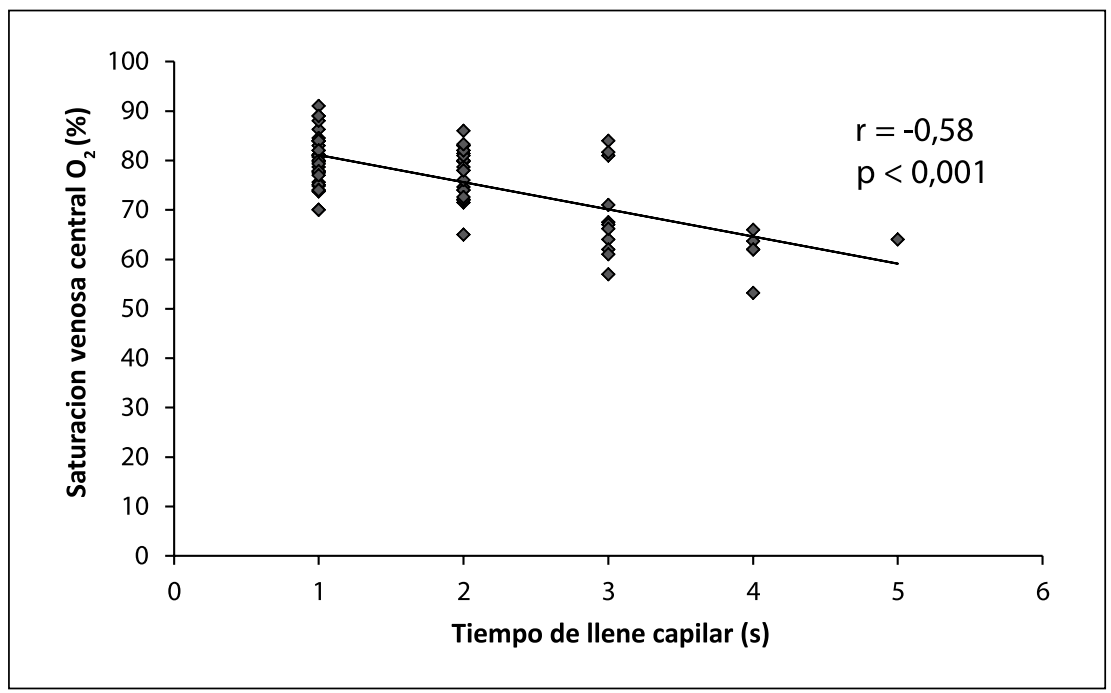

Figura 2. Correlación entre tiempo de llene capilar y saturación venosa central de oxígeno. trica de Spearman entre el tiempo de llenado del capilar y la saturación venosa fue de $-0,58$ $(\mathrm{p}<0,0001$, figura 2).

\section{Discusión}

Esta experiencia demuestra que el TLLC prolongado tiene una excelente capacidad de predicción de $\mathrm{ScvO}_{2}$ disminuida, en niños ingresados las primeras $24 \mathrm{~h}$ en UCI.

Raimer y cols., realizaron 39 mediciones de $\mathrm{ScvO}_{2}$ y TLLC, en esternón o frente (central) y en talón o dedos de extremidades inferiores (periférico), en veintiún niños, 12 con shock séptico, en los que se encontró resultados similares a los nuestros, donde un TLLC $<2$ s se asoció a $\mathrm{ScvO}_{2}>70 \%$. En esta experiencia el TLLC determinado en región central presentó un valor predictivo superior a la toma en zonas periféricas. También el TLLC presentó una correlación inversa con la $\mathrm{ScvO}_{2}$, con un $\mathrm{r}=-0,66$ central y de $-0,43$ para la determinación periférica ${ }^{8}$.

Nos llama la atención que los valores de la $\mathrm{ScvO}_{2}$ encontrados en nuestros pacientes fueron notoriamente más elevados de los valores observados en el estudio de Rivers, pero se 
asemejan a los reportados por otros autores en pacientes adultos ingresados a la $\mathrm{UCI}^{9-10}$. Lo anterior podría ser atribuido al diseño de nuestro estudio, ya que no fue realizado en sala de emergencias y no todas las mediciones fueron realizadas en las primeras horas de ingreso de los pacientes. El alto porcentaje de pacientes intubados y en ventilación mecánica durante la medición de la $\mathrm{ScvO}_{2}$ en nuestra serie puede haber contribuido a estos valores de $\mathrm{ScvO}_{2}$. Hernández y cols., demostró previamente que la maniobra de intubación puede aumentar significativamente los valores de la $\mathrm{ScvO}_{2}{ }^{11}$.

Nuestros hallazgos son consistentes con lo sugerido por Carcillo, en términos de que el uso de parámetros clínicos de fácil obtención como el TLLC, la frecuencia cardíaca y la presión arterial sistólica, pueden ser usados en atención primaria o en la sala de urgencias para identificar aquellos niños con riesgo de mortalidad y morbilidad funcional causadas por shock. En este estudio de 5.000 niños transferidos a cinco hospitales de alta complejidad, el uso adecuado de las guías del PALS, es decir normalización precoz del llene capilar a un valor de $\leq 2 \mathrm{~s}$ y de la presión arterial, resultó en una reducción de 2 veces en la mortalidad $y$ la morbilidad funcional en estos niños ${ }^{12}$. Estos resultados fueron muy similares al reporte de Han y cols., quien encontró que cada hora de retraso en el uso de las guías PALS en la sala de urgencias se asoció con un aumento del $40 \%$ en la mortalidad en niños con shock séptico ${ }^{13}$.

Otros autores, han planteado la necesidad de encontrar métodos más simples que la medición de la $\mathrm{ScvO}_{2}$ como meta en la reanimación inicial de la sepsis. Jones y cols., en un estudio multicéntrico de 300 adultos con sepsis severa o shock séptico, compararon el aclaramiento de lactato con la normalización de la $\mathrm{ScvO}_{2}$ como meta de resucitación. Se encontró que ambas estrategias fueron equivalentes, ya que la mortalidad hospitalaria fue similar en ambos grupos ${ }^{14}$. En esta misma línea, otro estudio multicéntrico de reciente aparición, también realizado en unidades de emergencia, sugiere que el empleo de protocolos de reanimación que incorporen la medición de $\mathrm{ScvO}_{2}$ no mejora el desenlace de pacientes adultos con shock séptico al compararlos con pacientes tratados sin la determinación de $\mathrm{ScvO}_{2} \mathrm{O}$ terapia estándar ${ }^{15}$.

A pesar de que su medición es afectada por múltiples factores externos y existe una gran variabilidad en la realización e interpretación del TLLC por el personal de salud, se ha demostrado que su determinación constituye uno de los signos clínicos más útiles en el diagnóstico de deshidratación en niños ${ }^{16,17}$.

El uso del TLLC como herramienta clínica ha sido evaluado en niños con deshidratación severa, una causa frecuente de shock en pediatría. Saavedra y cols., demostró que el llene capilar se correlaciona con el grado de deshidratación y un TLLC $>3 \mathrm{~s}$ indicaría un déficit de fluidos mayor a $100 \mathrm{ml} \cdot \mathrm{kg}^{18}$. También, se ha establecido que en niños con malaria grave, un TLLC $>2 \mathrm{~s}$ es un predictor independiente de mortalidad y es altamente específico para detectar infecciones severas en unidades de emergencia pediátrica ${ }^{19-21}$.

Algunas limitaciones de nuestro estudio la constituyen el hecho de que fue realizado en un solo centro, con pocos pacientes incluidos. Por escasez de personal, el llene capilar fue realizado por la enfermera a cargo del mismo paciente, lo que ciertamente puede introducir un sesgo. Este sesgo se atenúa si el llene capilar fue medido antes de tener el resultado del gas. No se evaluó la diferencia inter-observador para el TLLC previo al estudio.

Las variables hemoglobina y $\mathrm{T}^{\circ}$, no afectaron nuestros resultados, ya que excluimos pacientes con anemia y fiebre al momento de la evaluación. Sin embargo, otros factores que influyen en la medición del TLLC, como las condiciones de iluminación y $\mathrm{T}^{\mathrm{o}}$ ambiental, no fueron controladas.

El diseño de nuestro estudio no permitió investigar los cambios temporales del llene capilar en nuestros pacientes, por lo que en el futuro se debiera determinar si la normalización del TLLC tendría un impacto en el desenlace.

Se debe hacer énfasis que estos resultados no pueden extrapolarse a mediciones en el territorio de la vena cava inferior, frecuentemente utilizados en pediatría. Por otro lado, se debe recordar que el llene capilar es una medición de la perfusión del lecho capilar distal, 
por lo que no es equivalente a otras variables hemodinámicas sistémicas.

En la medida que el beneficio real de la medición continua o intermitente de la $\mathrm{ScvO}_{2}$ siga siendo motivo de controversia, creemos que la inspección del TLLC es un procedimiento seguro, no invasivo y fácil de realizar al lado de la cama del paciente, permitiendo al clínico identificar aquellos pacientes con hipoperfusión antes de continuar con procedimientos más invasivos.

En conclusión, la prolongación del TLLC $>2$ s es predictor de $\mathrm{ScvO}_{2}<70 \%$ en niños ingresados a UCI lo que apoya las recomendaciones del PALS. Este hallazgo, podría ser relevante en unidades de emergencia o en un escenario hospitalario fuera de la UCI, donde el uso de CVC es limitado y la $\mathrm{ScvO}_{2}$ no está disponible. La normalización del TLLC es una meta clínica razonable cuando la inserción de un CVC no sea posible.

Potenciales conflictos de interés: Este trabajo cumple con los requisitos sobre consentimiento /asentimiento informado, comité de ética, financiamiento, estudios animales y sobre la ausencia de conflictos de intereses según corresponda.

\section{Referencias}

1.- Rivers E, Nguyen B, Havstad S, et al: Early Goal-Directed Therapy Collaborative Group: Early goal-directed therapy in the treatment of severe sepsis and septic shock. N Engl J Med 2001; 345: 1368-77.

2.- Dellinger RP, Levy MM, Rhodes A, et al: Surviving sepsis campaign: international guidelines for management of severe sepsis and septic shock: 2012.Crit Care Med 2013; 41: 580-637.

3.- Carlbom DJ, Rubenfeld GD: Barriers to implementing protocol-based sepsis resuscitation in the emergency department-results of a national survey. Crit Care Med 2007; 35: 2525-32.

4.- Oliveira CF, Nogueira de Sá FR, Oliveira DS, et al: Time- and fluid-sensitive resuscitation for hemodynamic support of children in septic shock: barriers to the implementation of the American College of Critical Care Medicine/Pediatric Advanced Life Support Guidelines in a pediatric intensive care unit in a developing world. Pediatr Emerg Care 2008; 24: 810-5.
5.- Inwald DP, Tasker R, Peters MJ, Nadel S: Emergency management of children with severe sepsis in the United Kingdom: The result of the Paediatric Intensive Care Society sepsis audit. Arch Dis Child 2009; 94: 348-53.

6.- Hazinski MF, Zaritsky AL, Nadkarni VM, et al (Eds): PALS Provider Manual. Dallas, TX. American Heart Association, 2002.

7.- Strange $G R(E d)$ : APLS: The Pediatric Emergency Medicine Course. Elk Grove Village, IL, American Academy of Pediatrics and American College of Emergency Physicians, 1998.

8.- Raimer PL, Han YY, Weber MS, Annich GM, Custer JR: A Normal capillary refill time of $<2$ seconds is associated with superior vena cava oxygen saturation of $>70 \%$ J Pediatr 2011; 158: 968-72.

9.- Bracht H, Hänggi M, Jeker B, et al: Incidence of low central venous oxygen saturation during unplanned admissions in a multidisciplinary intensive care unit: an observational study. Crit Care 2007; $11 \mathrm{R} 2$.

10.- Van Beest PA, Hofstra HH, Schultz MJ, et al: The incidence of low venous oxygen saturation on admission in the UCI: a multicenter observational study in the Netherland. Crit Care 2008; 12: R33.

11.- Hernández $G$, Peña H, Cornejo $R$, et al: Impact of emergency intubation on central venous oxygen saturation in critically ill patients: a multicenter observational study. Crit Care 2009; 13: R63.

12.- Carcillo JA, Kuch BA, Han YY, et al: Mortality and functional morbidity after use of PALS/APLS by community physicians. Pediatrics 2009; 124: 500-8.

13.- Han YY, Carcillo JA, Dragotta MA, et al: Early reversal of pediatric-neonatal septic shock by community physicians is associated with improved outcome. Pediatrics 2003; 112: 793-9.

14.- Jones AE, Shapiro NI, Trzeciak S, Arnold RC, Claremont HA, Kline JA: Emergency Medicine Shock Research Network (EMShockNet) Investigators. Lactate clearance $v s$ central venous oxygen saturation as goals of early sepsis therapy: a randomized clinical trial. JAMA 2010; 303: 739-46.

15.- The ProCESS Investigators. A Randomized Trial of Protocol-Based Care for Early Septic Shock. NEJM 2014; 370: 1683-93

16.- Lobos AT, Menon KA: Multidisciplinary survey on capillary refill time: Inconsistent performance and interpretation of a common clinical test. Pediatr Crit Care Med 2008; 9: 386-91.

17.- Pickard A, Karlen W, Ansermino JM: Capillary refill time: is it still a useful clinical sign? Anesth Analg 2011; 113: $120-3$. 
18.- Saavedra JM, Harris GD, Li S, Finberg L: Capillary refilling (skin turgor) in the assessment of dehydration. Am J Dis Child 1991; 145: 296-8.

19.- Steiner MJ, De Walt DA, Byerley JS: Is This Child Dehydrated? JAMA 2004; 291: 2746-54.

20.- Evans JA, May J, Ansong D, et al: Capillary refill time as an independent prognostic indicator in severe and complicated malaria. J Pediatr 2006; 149: 676-81.

21.- Thompson M, Coad N, Harnden A, Mayon-White R, Perera $R$, Mant $D$ : How well do vital signs identify children with serious infections in paediatric emergency care? Arch Dis Child 2009; 94: 888-93. 